tal, Universität Vechta), Österreich (Universität Wien), Russland (Baltische Föderale Immanuel-Kant-Universität zu Kaliningrad), Holland (Universität Nijmegen), England (Universität Nottingham) und Italien (Universität Napoli).

Die Vorträge deckten ein breites und reiches Betrachtungsspektrum zu Werken sowohl der Inneren Emigranten als auch der Exilanten ab. Sie befassten sich mit historischen und theoretischen Aspekten der „Inneren Emigration“ und des Exils, setzten sich mit Einzelautoren auseinander (Stefan Andres, Oskar Loerke, Ernst Wiechert, Eugen Kogon, Walter Poller, Ernst Jünger, Max Picard, Thomas Mann, Erich Kästner, Hermann Stehr, Johannes Bobrowski), schilderten eine besondere Lage der österreichischen Schriftsteller unter der NSHerrschaft (Erika Mitterer, Heimito von Doderer, Bruno Heilig) und zeigten die Situation der Literaten jenseits der Grenzen und nach der Rückkehr auf (Georg Lukács, Joseph Roth, Hermann Hesse, Bertolt Brecht, Irmgard Keun).

In ihrer Ansprache zur Eröffnung der Tagung verwies die Prodekanin des Fachbereichs für Neophilologie, Frau Prof. Mikołajczyk, auf den internationalen und nationalen Aspekt der Tagung, denn sie wurde von zwei polnischen, zwei deutschen und einer russischen Universität zusammen mit der Internationalen Ernst-Wiechert-Gesellschaft organisiert. Herr Prof. Karolak betonte, dass die Konferenz nicht nur den Gedankenaustausch namhafter europäischer Forscher fördern, sondern zugleich auch Nachwuchswissenschaftler zur wissenschaftlichen Beschäftigung mit dem Werk der einst bekannten, heutzutage fast komplett in Vergessenheit geratenen Schriftsteller und Dichter der „Inneren Emigration“ anregen solle.

Für den Herbst 2015 ist die Publikation in der Schriftenreihe der Internationalen ErnstWiechert-Gesellschaft beim de Gruyter Verlag geplant.

Beeindruckend an der Tagung war nicht nur die entspannte und seitens der Teilnehmer freundliche Arbeitsatmosphäre, sondern zugleich auch die Aufgeschlossenheit, die gleichermaßen zum Erfolg der Tagung beitrug. Der Wunsch der Veranstalter, der Tagung einen zyklischen Charakter zu verleihen, wurde von den Konferenzteilnehmern sehr begrüßt.

Marcin Golaszewski*

\title{
„HOMO MYTHICUS” MYTHISCHE IDENTITÄTSMUSTER. \\ VI. Internationale Interdisziplinäre Wissenschaftliche Tagung aus der Reihe „Das Phantastische und das Wunderbare“ Zielona Góra, 27-28. März 2012
}

Die gegenwärtige Kultur ist nach wie vor von mythischen Ästhetisierungsmustern in allen denkbaren Kunstformen umstellt. Bei näherer Untersuchung der Präsenz des Mythos drängt sich nicht die Frage nach den Ausdrucksmöglichkeiten mythischer Überlieferungen,

\footnotetext{
* Der Verfasser des Tagungsberichtes ist wissenschaftlicher Mitarbeiter im Institut für Germanistik an der Adam-Mickiewicz-Universität in Poznań im Rahmen des Förderprogrammes des Polnischen Nationalen Forschungszentrums (NCN): FUGA (2013-2016). Die Publikation entstand dank finanzieller Unterstützung des Polnischen Nationalen Forschungszentrums (NCN). Vertragsnummer: 2013/08/S/ HS2/00224.
} 
sondern nach deren Quellen und den zu erfüllenden Funktionen im Bewusstsein des postmodernen Rezipienten [auf]. Sowohl Neu-Narrationen, als auch Neu-Interpretationen des Mythos untermauern seine Attraktivität in der Gegenwartskultur und belegen trotz aller Degradierungsprozesse seinen großen Einfluss auf die Kulturerzeugnisse in allen Bereichen. Das Fragewörterpaar WARUM und WIE war den Organisatoren der VI. Internationalen Wissenschaftlichen Konferenz, dem Institut für Polnische Philologie sowie dem Lehrstuhl für Mythopoetik und Philosophie der Literatur besonders wichtig. Verantwortlich für die Veranstaltung waren Prof. Dr. Bogdan Trocha, Dr. Paweł Wałowski sowie Mag. Kateryna Atamanova. Die Konferenz wurde am 27. März von den zur Tagung eingeladenen Vertretern der Universitätsleitung, dem Dekan der Humanistischen Fakultät, Prof. Dr. habil. Wojciech Strzyżewski eröffnet. Der Einladung folgten auch Literatur-, Kulturwissenschaftler und Mitarbeiter des Instituts für Philosophie der Universität Zielona Góra. An drei Tagen gab es insgesamt 34 Vorträge von Referentinnen und Referenten aus Deutschland (Köln), Österreich (Wien), Russland (Sankt Petersburg), der Ukraine (Schytomyr) und polnischen Universitäten und Hochschulen (Katowice, Kielce, Łódź, Opole, Poznań, Racibórz, Słupsk, Szczecin, Warszawa, Wrocław, Zielona Góra). In den Vorträgen wurde ein reiches Betrachtungsspektrum zu mythischen Identitätsmustern abgedeckt. Die Vortragenden befassten sich nicht nur mit spezifischen Bezügen zum Mythos in den analysierten Werken sowohl der „hohen“, als auch der Unterhaltungsliteratur, sondern auch mit dem theoretischen Reservoir von T. Adorno, J. Assmann, R. Barthes, E. Cassirer, C.G. Jung, T. Mann, L. Kołakowski u.a.

Die Plenartagung wurde mit einem Beitrag von NATALIA SZEWCZENKO (Sankt Petersburg) eingeleitet, die sich der Problematik des Mythischen und Rationellen in der Symbolsprache der Wissenschaft in statu nascendi zuwandte. Die Funktionen christlich-barocker und expressionistischer Mythosgestalten wurden zum Gegenstand der kritischen Analyse im Beitrag von ANDREA RUDOLPH (Opole), die am Beispiel des Nachkriegswerks Ilse Langners den ästhetischen und kognitiven Wert der mythischen Figuren sowie deren Erscheinungsbilder bergründete. In ihren Untersuchungen ging sie auch auf die sprachlichen Aspekte und Mittel in Langners Schaffen ein und wies auf eine von der Autorin bevorzugt angewendete Modellierung der Wirklichkeit zum Symbolischen hin. Die Referentin zeigte ebenfalls ein Modell auf, dank dem Sätzen und Dialogen aufgrund der eingesetzten Allegorien besonderer Raum und Tiefe verliehen wurden. ANDRZEJ DRAGULA (Szczecin) lenkte sein Augenmerk auf die mythopoetischen Gedankenkonstrukte des gegenwärtigen ,homo mythicus“, kontrastiv dargestellt zur Geistigkeit des „homo religiosus“ in der Postmoderne. Der Beitrag von Dragula, dem eine Diskussionsrunde folgte, schloss die Plenartagung ab. Im weiteren Verlauf der Konferenz war die Tagung in Sektionen A und B vorgesehen. Die Einteilung erfolgte nach sprachlichen Gesichtspunkten; die Tagungssprache der Sektion A war Deutsch und Polnisch und die Sektion B tagte auf Russisch und Polnisch.

Interessante Beträge lieferten die Vortragenden in Sektion A. Den zweiten Teil der Tagung leitete als erste ARLETTA SZMORHUN (Zielona Góra) mit dem Beitrag „Paradiesische Metamorphosen bei Dagmar Nick, Leonie Ossowski und Ingo Schulze“ ein, in dem sie auf die Bibel als maßgebendes Werk mit Fragen nach kultureller Selbstverständigung einging. Die Referentin versuchte, das Ausverhandeln von Geschlechterrollen herauszuarbeiten und warf ein neues Licht auf das Schaffen von Dagmar Nick. Mit großer Sorgfalt untersuchte die Vortragende die Vertreibungsgeschichte bei Ossowski und Schulze, bei denen sie theologische Zusammenhänge mit diversen religiösen Handlungs- und Denkmodellen konstatierte. ADAM SOBEK 
(Poznań) beschäftigte sich in seinem Beitrag mit einer Auslegung des Mythos in Kategorien der Bewertung oder Verhässlichung der Realität am Beispiel ausgewählter Werke von Otto F. Walter. Grundlage seiner Auseinandersetzung war das nach Cassirer zitierte Model, in dem der deutsche Philosoph den Mythos als die vorwissenschaftliche Welterklärung deutete. In den besprochenen Werken versuchte der Referent die Bezüge nicht nur zu griechischen Mythen, sondern auch zu altgermanischen Sagen mit speziellem Fokus auf deren Funktionscharakter in fiktionalen Texten aufzuzeigen. Ins Zentrum seiner Überlegungen wurde das Anliegen der Entlarvung sowie der Dekonstruktion des Mythenhaften gerückt. ALEXANDER RIHA (Wien) stellte in seinem Vortrag über Hauptmanns „utopischen“ Roman Die Insel der Großen Mutter die Sprengung der Genregrenzen durch die (Re-)Sakralisierung bestimmter Aspekte scharfsinnig dar. Außerdem analysierte der Referent pointiert den Text, der den Welterklärungsmodellen generell zu misstrauen schien und damit ein Bild des ausbrechenden Zweiten Weltkriegs parabelartig in der Realität vorwegnahm. Der Vortrag von PAWEŁ WAŁOWSKI (Zielona Góra) war der neuesten DDR-Prosa und einer besonderen Erörterung der mythischen Perspektive als Interpretationsdominante gewidmet. Er versuchte seine in der Einleitung aufgestellten Thesen anhand K. Gawlittas Ost-Westpassagen (2010), M. Kumpfmüllers Hampels Fluchten (2000) und A. C. Voorhoeves Lilly und den Linden (2004) zu beweisen. Wałowski diskutierte in seinem Vortrag ebenso die verschiedenen Positionen von Ästhetisierungsprozessen des Topos DDR als Schauplatz menschlicher Schicksale und wies auf das vom Themenkomplex demythisierte Bild der DDR hin. Die Überlegungen des Referenten schlossen damit auch den letzten Teil der Tagungen am ersten Konferenztag ab. Zu Beginn des zweiten Konferenztags stellte AGNIESZKA DYLEWSKA (Zielona Góra) ihre Reflexionen über den Mythos und das Mythische in Botho Strauß Roman Vom Aufenthalt vor. Dabei setze sie sich mit der Begriffsbestimmung des Mythos und dem interdisziplinären Mythosdiskurs auseinander. Die Referentin ging detailliert auf die Zeitauffassung des Mythischen ein und stellte antike Motive, mythische Orte und biblische Anspielungen im analysierten Werk heraus. Ludwig II. und die Arbeit am Mythos in Klaus Manns Vergittertes Fenster bildeten den Gegenstand des Referats von ROLF FÜLLMANN (Köln): Der Referent bezog sich zu Beginn des Vortrags auf Paul Verlaines Gedicht A Luis II de Bavière aus dem Jahr 1886, um die Wirkung einer Arbeit am eigenen Mythos zu demonstrieren. Füllmanns Schlussfolgerungen, aus der Novelle steige ein neuer Mythos und ein neuer ,Homo mythicus` auf und der tote König könne seinen Nimbus überzeitlich und übernational entfalten, schlossen sein Referat ab. Der Vortrag von ELIGIUSZ PIOTROWSKI (Zielona Góra-Szczecin) unterzog die Film-Trilogie Matrix einer eingehenden Analyse und präsentierte den Film als Ort der Vertiefung theologischer Reflexion. Gegenstand seiner kontrastiven Erörterung war auch der jüdisch-christliche Kontext mit sowohl absichtlichen, als auch zufälligen Andeutungen. Abschließend griff der Referent die Botschaft der Erlösung im anderen Klassiker, der Trilogie J.R.R Tolkiens und dem Kultfilm Der Herr der Ringe auf. Der Vortrag bildete den Abschluss in Sektion A. Zum Schluss bedankten sich die Organisatoren der ertragreichen Konferenz bei allen Teilnehmern und Gästen für ihre zahlreichen Wortmeldungen und aufschlussreichen Diskussionsbeiträge recht herzlich und kündigten zugleich das Vorhaben der Herausgabe von Beiträgen in einem Sammelband an. Außerdem luden sie die Gäste zur Teilnahme an der nächsten Konferenz im Zyklus Das Phantastische und das Wunderbare im Jahr 2014 ein, die in Zusammenarbeit mit der Adam-Mickiewicz-Universität Poznań veranstaltet wird. 



\section{ANGABEN ZU DEN AUTOREN}

Prof. UAM dr hab. BEATE SOMMERFELD Instytut Filologii Germańskiej

Uniwersytet im. Adama Mickiewicza w Poznaniu

al. Niepodległości 4

61-874 Poznań

e-mail: ifguam@amu.edu.pl

dr KAROLINA KĘSICKA

Instytut Filologii Germańskiej

Uniwersytet im. Adama Mickiewicza w Poznaniu

al. Niepodległości 4

61-874 Poznań

e-mail: ifguam@amu.edu.pl

dr KALINA KUPCZYŃSKA

Instytut Filologii Germańskiej

Uniwersytet Łódzki

ul. Pomorska 171/173

90-236 Łódź

e-mail: mariawisniewska@uni.lodz.pl

dr ELISABETH HOLLERWEGER

Germanistisches Seminar der Philosophischen Fakultät

Universität Siegen

Adolf-Reichwein-Straße 2

57076 Siegen

e-mail: hollerweger@germanistik.uni-siegen.de

dr MAŁgORZATA KoryCiŃSKA-WEGNER

Instytut Filologii Germańskiej

Uniwersytet im. Adama Mickiewicza w Poznaniu

al. Niepodległości 4

61-874 Poznań

e-mail: ifguam@amu.edu.pl 
Projekt okładki: Ewa Wąsowska

Redaktor prowadzący: Katarzyna Muzia

Redaktor techniczny: Elżbieta Rygielska

WYDAWNICTWO NAUKOWE UNIWERSYTETU IM. ADAMA MICKIEWICZA W POZNANIU 61-701 POZNAŃ, UL. FREDRY 10 www.press.amu.edu.pl

Sekretariat: tel. 6182946 46, faks 6182946 47, e-mail: wydnauk@amu.edu.pl Dział sprzedaży: tel. 6182946 40, e-mail: press@amu.edu.pl Ark. wyd. 9,25. Ark. druk. 7,75.

DRUK I OPRAWA: EXPOL, WŁOCŁAWEK, UL. BRZESKA 4 